\title{
Prácticas parentales: un estudio comparado en adolescentes portugueses y españoles*
}

\section{Parental practices: a comparative study of Portuguese and Spanish} adolescents

Recepción: 19 Octubre 2017 | Aceptación: 24 Octubre 2019

\author{
Santiago Mendo LÁZaro \\ Universidad de Extremadura, España \\ ORCID: http://orcid.org/0000-0002-4377-8416 \\ María-Isabel Polo Del Río \\ Universidad de Extremadura, España \\ ORCID: http://orcid.org/0000-0001-7107-1330 \\ Fernando Fajardo Bullón \\ Universidad de Extremadura, España \\ ORCID: http://orcid.org/0000-0003-0198-0170 \\ Elena Felipe Castaño \\ Universidad de Extremadura, España \\ ORCID: http://orcid.org/0000-0001-6167-877X \\ Benito León Del Barco \\ Universidad de Extremadura, España \\ ORCID: http://orcid.org/0000-0003-0061-9498
}

a Autor de correspondencia. Correo electrónico: smendo@unex.es

Para citar este artículo: Mendo Lázaro, S., Polo del Río, M. I., Fajardo Bullón, F., Felipe Castaño, E., \& León del Barco, B. (2019). Prácticas parentales: un estudio comparado en adolescentes portugueses y españoles. Universitas Psychologica, 18(4), 1-14. https://doi.org/1 0.11144/Javeriana.upsy18-4.ppec

\section{RESUMEN}

El objetivo del presente trabajo es estudiar las diferencias en la percepción de los adolescentes sobre el afecto y la crítica que manifiestan los padres hacia sus hijos, y la forma en que establecen y exigen el cumplimiento de las normas, en función de la nacionalidad (portuguesa y española) y el género de los adolescentes. También analizamos la influencia del estilo parental sobre el afecto y la comunicación que perciben de sus padres. Participaron 1260 adolescentes (674 portugueses y 531 españoles) de entre 12 y 16 años $(M=13.98, D T=1.3)$. Los datos se obtuvieron a través de autoinformes que evalúan la percepción del Afecto-Comunicación, la Crítica-Rechazo (EA-H) y las formas (Inductiva, Rígida e Indulgente) que tienen los padres de establecer y exigir el cumplimiento de las normas (ENE-H). Utilizando un diseño transversal, y mediante análisis multivariados, de medidas repetidas y de regresión, se confirma la existencia de diferencias interculturales e intraculturales en la percepción del Afecto-comunicación, critica-rechazo y el estilo parental, y que la forma Inductiva es un fuerte predictor del afecto y cariño de los progenitores españoles y portugueses.

Palabras clave

prácticas parentales; afecto; comunicación; crítica; rechazo; normas.

\section{ABSTRACT}

The aim of the present work is to study differences in the adolescents' perception their affection and criticism shown by their parents and the way their parents establish and enforce rules, according to nationality (Portuguese and Spanish), adolescents' gender, and the interaction of 
the adolescents' nationality/gender. A total of 1260 adolescents (674 Portuguese and 531 Spanish) between 12 and 16 years old $(M=13.98, S D=1.3)$ participated. The data were obtained through self-reports that evaluate the perception of Affect-Communication and CriticismRejection (EA-H) and the forms (inductive, rigid and indulgent) that parents have to establish and demand compliance with the norms (ENE-H). Using a crosssectional design, and through multivariate analysis of repeated and regression measures, we confirm: the existence of intercultural and intracultural differences in the perception of affection-communication, criticismrejection, and parental style. That the inductive form is a strong predictor of the affection of the Spanish and Portuguese parents.

Keywords

parental practices; affection; communication; criticism; rejection; rules.

Los padres constituyen el principal medio de socialización y desarrollo de toda persona desde muy temprana edad. Es indiscutible el papel de la familia en el desarrollo psicosocial, y los padres son la fuerza más poderosa en la vida de sus hijos. La familia es el contexto donde se reciben los primeros mensajes de cariño, aceptación, rechazo o abandono (Rohner, 1975). Para Rodrigo y Palacios (1998), las concepciones de los padres sobre la educación, las relaciones interpersonales y los estilos de crianza son factores determinantes para el desarrollo de los hijos. Los estilos parentales han sido utilizados para explicar los efectos de la socialización familiar sobre la competencia de los hijos. Para que se dé un desarrollo infantil óptimo, debe existir un buen nivel afectivo, combinado con un alto nivel de demandas y control parental (Aroca \& Cánovas, 2012).

Para Rohner y Carrasco (2014), la teoría de la aceptación-rechazo parental — sustentada en la investigación - trata de explicar y predecir las causas, consecuencias y correlatos de la aceptación-rechazo a lo largo del desarrollo humano. Según Rohner (1975), la aceptación-rechazo parental supone un continuo donde, por un lado, están los padres que muestran a sus hijos amor y afecto tanto verbal, como físicamente. Y, por otro lado, se encuentran los padres que sienten aversión hacia sus hijos, les critican y les rechazan.

Particularmente influyente en el estudio de los estilos parentales ha sido el aporte de Baumrind (1971), quien delineó diferentes estilos parentales que hoy son ampliamente conocidos en la literatura científica (estilos). Más tarde, Baumrind (1996) define los estilos parentales en función de la combinación de dos factores generales, que denomina "exigencia" y "sensibilidad-receptividad", aunque la mayoría de los estudios se ajustan al modelo de Maccoby y Martin (1983), que clasifica a los padres en cuatro estilos a partir de dos dimensiones (afecto y control). Juntos, los cuatro estilos proporcionan un marco conceptual importante para un conjunto de conductas parentales y metas de crianza de los hijos (Domenech, Donovick, \& Crowley, 2009).

Se pueden clasificar las investigaciones sobre los estilos parentales en dos grupos. Por un lado, un primer grupo mayoritario de investigaciones que se ha centrado en la influencia que estos ejercen sobre el desarrollo y la socialización de niños y adolescentes (García, Serra, Zacarés, \& García, 2018; López-Soler, Puerto, LópezPina, \& Prieto, 2009; Rodrigues, Veiga, Fuentes, \& García, 2013). Otras investigaciones señalan las consecuencias positivas del estilo parental centrado en el amor y el control positivo (Alegre, 2011; Grolnick \& Pomerantz, 2009; Nunes, Bodden, Lemos, Lorence, \& Jiménez, 2014; Torío, Peña, \& Inda, 2008; Tur-Porcar, Mestre, Samper, \& Malonda, 2012). La afectividad positiva y la comunicación parental previenen conductas desajustadas en los adolescentes, estimulan el desarrollo positivo (Gracia, Lila, \& García, 2008; Mansager \& Volk, 2004; Sánchez-Sandoval, 2002) y constituyen un requisito preventivo para la salud mental (León, Fajardo, Mendo, Rasskin, \& Iglesias, 2018). Por otro lado, niveles inadecuados de afecto, de apoyo y predominio de la agresión y el rechazo hacia los hijos generan consecuencias negativas (Mestre, Tur, Samper, \& Latorre, 2010; Torío, Peña, \& Inda, 2008; Gracia et al., 2008; Oliva, Parra, \& Arranz, 2008; Rudy \& Grusec, 2006; Martínez, Fuertes, Ramos, \& 
Hernández, 2003), y se corresponden con la manifestación de problemas conductuales de agresividad, hostilidad y delincuencia (León, Felipe, Polo, \& Fajardo, 2015; Moreno, Estévez, Jiménez, \& Murgui, 2018; Muris, Meesters, Morren, \& Moorman, 2004).

Ciertos estudios comparan el estilo educativo del padre y la madre de forma separada, con el fin de establecer cuál es más influyente en el desarrollo de los hijos adolescentes, y determinar cuál es la percepción que ellos tienen de los estilos parentales. Entre las investigaciones más destacas se encuentra la de Oliva, Parra, SánchezQueija y López (2007), quienes concluyen que tanto los hijos adolescentes como las hijas conceden puntuaciones más altas a sus madres en cada una de las dimensiones del estilo educativo parental (afecto y comunicación, promoción de autonomía, control conductual, control psicológico de los sentimientos y sentido del humor). Laible y Carlo (2004) y TurPorcar et al. (2012) ponen de manifiesto que, independientemente del sexo de los hijos, las madres son más afectuosas y favorecen más la autonomía de los hijos.

Por otro lado, un segundo grupo minoritario en la investigación se ha centrado en analizar los factores que influyen en los estilos educativos parentales, como la edad, el sexo, el orden de nacimiento, número de hijos, el contexto sociocultural al que pertenecen, o el tiempo dedicado a los hijos o al trabajo.

En cuanto al sexo de los hijos, existen evidencias de que esta variable influye en las características del estilo parental percibido, tanto por el padre como por la madre. Los hijos varones muestran una percepción de crítica-rechazo por parte de sus padres, mayor que las hijas (Polo, Fajardo, Martín, Gómez, \& León, 2012; Bersabé, Fuentes, \& Motrico, 2001), y perciben más rechazo y crítica de su padre que de su madre (Motrico, Fuentes, \& Bersabé, 2001). Por otro lado, tanto los hijos como las hijas perciben más afecto y comunicación de la madre que del padre (Mendo, León, Polo, Yuste, \& López, 2019; Polo et al., 2012; Oliva et al., 2007).

Con respecto a los factores orden de nacimiento y número de hijos, Furman y Lanthier
(2002) afirman que son los hijos primogénitos y los hijos únicos quienes reciben más atención, comunicación y mayores cuidados parentales.

En relación con el tiempo dedicado a los hijos o al trabajo, las horas laborales de los padres se asocian con las prácticas de crianza de los hijos: el empleo de la madre se vincula a la conducta del niño, un mayor número de horas de trabajo remunerado se asocian con prácticas de crianza menos cálidas (Hadzic, Magee, \& Robinson, 2013).

En cuanto al contexto cultural, los estilos educativos parentales presentan diferentes significados e implicaciones para los adolescentes, dependiendo del contexto al que pertenecen (García \& Gracia, 2010; León, Mendo, Polo, \& López, 2019; Nunes et al., 2014). En contextos socioculturales anglosajones, el estilo parental autorizativo, caracterizado por la combinación de afecto e imposición (alta aceptación/implicación y alta severidad/imposición), es un estilo educativo adecuado (Fuentes, García, Gracia, \& Alarcón, 2015). Sin embargo, estudios realizados en otras culturas diferentes, como la cultura afroamericana (Baumrind, 1972), o la cultura árabe (Dwairy, 2008) concluyen que es el estilo autoritario es el apropiado.

En esta misma línea, un estudio realizado por Fuentes et al. (2015) establece que el estilo parental idóneo, en el caso de España, es el estilo indulgente, basado fundamentalmente en el afecto y no en la imposición parental. Coincidiendo con esto, Rodrigues et al. (2013) concluyen que el estilo indulgente (caracterizado por la aceptación/implicación, pero sin el componente de severidad/imposición) aparece en el contexto cultural portugués como el estilo parental óptimo.

Un problema relacionado con la investigación de los estilos parentales es que los padres no suelen tener un estilo de crianza definido. Aunque la mayoría de las investigaciones se ajustan al modelo de dos dimensiones de Maccoby y Martin (1983), existen otros basados en dimensiones como la promoción de la autonomía, la revelación y el humor, que habría que tener en cuenta en un modelo 
multidimensional de los estilos parentales (Oliva et al., 2008). Por esto, se considera oportuno utilizar las dimensiones Afecto-comunicación y Exigencias-control, lo que permite determinar en qué medida se relacionan las principales dimensiones de los estilos parentales.

En esta investigación se presenta un estudio comparativo entre dos poblaciones limítrofes, Extremadura, en España, y Alentejo, en Portugal, regiones vecinas y de similar nivel de desarrollo, que comparten muchos rasgos demográficos, geográficos (Instituto Nacional de Estadística, 2014), educativos (Huertas \& Rego, 2005) y ecológicos (Jiménez \& Fernández, 2009), con similares niveles de envejecimiento poblacional, bajas tasas de natalidad o tasas de desempleo superiores a las medias nacionales.

Aunque algunos estudios apuntan a una similitud en el ámbito familiar de los adolescentes portugueses y españoles (Serrano, El-Astal, \& Faro, 2004), los estilos parentales y el desarrollo de los hijos pueden tener consecuencias diferentes al relacionarlos con la cultura (Darling \& Steinberg, 1993).

En este sentido, Extremadura en España, y Alentejo en Portugal son el marco ideal para el análisis de diferencias con base en la cultura. La homogeneidad de estas regiones posibilita una comparación, que elimina — en parte- factores de tipo económico, demográfico, ambiental o geográfico.

Como primer objetivo del presente trabajo, se pretende estudiar las diferencias en la percepción de los adolescentes del afecto, interés y comunicación que manifiestan sus padres, y de la forma en que sus padres establecen y exigen el cumplimiento de las normas, en función de su nacionalidad (portuguesa y española) y género, así como las diferencias entre ambos progenitores. Como segundo objetivo, se analiza el efecto de las formas de establecer y exigir el cumplimiento de las normas de ambos progenitores, sobre el afecto y la comunicación que perciben los adolescentes españoles y portugueses de sus padres.

\section{Método}

\section{Participantes}

La muestra estuvo constituida por 1260 adolescentes (674 portugueses y 531 españoles) que viven en las comarcas del Alentejo y Extremadura. La edad media de los adolescentes portugueses fue 13.84 años $(D T=1.3$; rango: $12-16) ; 53.4 \%(n=360)$ mujeres y 43.6 $\%(n=314)$ varones. La edad media de los adolescentes españoles fue 13.98 años (DT = 1.38; rango: $12-17)$; el $43.1 \%(n=229)$ mujeres y el $56.9 \%(n=302)$ varones. El número de participantes se determinó a partir de la cantidad de alumnos matriculados en el curso 2009-2010, considerando un error muestral de $3 \%$ y un nivel de confianza de $95.5 \%$.

La selección de los estudiantes portugueses y españoles se realizó mediante un muestreo polietápico estratificado por conglomerados y selección aleatoria de los grupos en los centros que disponían de varias líneas en los cursos de educación secundaria.

\section{Instrumentos de medida}

\section{Información sociodemográfica}

Se elaboró un cuestionario ad hoc para recoger información referente a la nacionalidad, edad y sexo de los adolescentes, el número de hermanos, así como el lugar que ocupan entre ellos, y con quién conviven (ambos progenitores, el padre, la madre u otro familiar o tutor).

Escala de Afecto versión hijos (EA-H, Bersabé et al., 2001)

Se compone de dos factores, cada uno consta de 10 ítems que se presentan en formato Likert, con cinco grados de frecuencia que representan un continuo que va desde "nunca" hasta "siempre". El primer factor llamado Afectocomunicación evalúa la percepción que tienen los hijos del afecto, el interés y comunicación que 
manifiestan sus padres (padre-madre) hacia ellos: "me consuela cuando estoy triste", "me acepta tal y como soy", "es cariñoso/a conmigo". Presenta un alfa de Cronbach para la modalidad padre de 0.92 , y de 0.79 para la modalidad madre. El segundo factor llamado Crítica-rechazo evalúa la crítica, el rechazo y la falta de confianza de los padres (padre-madre) hacia sus hijos: "lo que hago le parece mal", "está a disgusto cuando yo estoy en casa", "le gustaría que fuera diferente". Presenta un alfa de Cronbach para la modalidad padre de 0.83 y de 0.82 para la modalidad madre. El rango de puntuación de cada factor está comprendido entre 10 y 50 .

Escala de Normas y Exigencias versión hijos (ENE-H, Bersabé et al., 2001)

Consta de 3 factores: F1 Forma Inductiva, F2 Forma Rígida y F3 Forma Indulgente, que tienen los padres de establecer y exigir el cumplimiento de las normas. El primer y segundo factor lo componen 10 ítems, F1 Padre $(\alpha=0.79) F 1_{\text {Madre }}(\alpha$ $=0.76) ; \mathrm{F} 2_{\text {Padre }}(\alpha=0.77) \mathrm{F} 2_{\text {Madre }}(\alpha=0.76), \mathrm{y}$ el tercero 8 ítems, F3 Padre $(\alpha=0.73) \mathrm{F}_{\text {Madre }}(\alpha$ $=0.74)$. La escala de respuesta es la misma que en la EA-H, el rango de puntuación de los dos primeros factores está comprendido entre 10 y 50 , y del tercer factor entre 8 y 40 . El adolescente debe responder al contenido de cada ítem según la percepción que tiene del estilo educativo de su padre y de su madre (por ejemplo, "antes de castigarme, escucha mis razones").

Bersabé et al. (2001) construyen las escalas Afecto y Normas y Exigencias para evaluar los estilos educativos parentales, centradas en las relaciones entre padres e hijos en la etapa en la que estos son adolescentes, teniendo en cuenta la perspectiva de ambos. ¿Por qué nos interesa la percepción de los hijos sobre los estilos parentales? Diversos estudios encuentran baja coincidencia entre las opiniones de los padres e hijos sobre las prácticas parentales (Bersabé et al., 2001; González \& Landero, 2012). Los padres suelen tener una percepción propia sobre sus prácticas parentales, a veces sesgada por una deseabilidad social. La percepción de los hijos adolescentes tiene menos sesgo, es más objetiva y puede ser un importante predictor de sus respuestas, más que la de los padres.

\section{Procedimiento}

Los datos se obtuvieron a partir de ocho escuelas públicas, cuatro del Alentejo (Portugal) y cuatro de Extremadura (España), seleccionadas por muestreo simple cúmulo de todos los centros educativos. Los consejos escolares de todos los centros acordaron participar. Se solicitaron formularios de consentimiento informado a los padres y adolescentes. Los instrumentos se completaron ante un entrevistador entrenado y en el contexto del aula, con una duración de entre 20 y 25 minutos. Su participación fue voluntaria, y no se ofreció compensación por su participación.

\section{Análisis estadísticos}

Los análisis estadísticos se llevaron a cabo con el paquete SPSS v-21. Se realizaron análisis exploratorios de los datos para detectar valores perdidos y casos atípicos, así como determinar el cumplimiento de los supuestos de normalidad, homocedasticidad, linealidad, y homogeneidad de varianzas, además se siguieron los procedimientos recomendados por Tabachnick y Fidell (2007) para examinar el cumplimiento de presupuestos multivariados, donde se obtuvieron resultados satisfactorios en todos los casos. $\mathrm{Se}$ examinaron posibles diferencias en las características sociodemográficas (convivencia, número de hermanos, edad y género) de ambos países, previamente al análisis de las diferencias en las prácticas parentales, en función de la nacionalidad de los adolescentes. Por último, se realizaron Manova y $t$ de Student para las subescalas del EA-H (afecto) y ENE-H (prácticas parentales), así como análisis de regresión lineal múltiple en los que las variables dependientes fueron Afectocomunicación padre/madre,donde las variables predictoras son las prácticas parentales (Forma Inductiva/Rígida/Indulgente padre y madre). 


\section{Resultados}

\section{Resultados sociodemográficos}

En primer lugar, se presenta la comparación con base en la nacionalidad de algunas de las principales características sociodemográficas correlacionadas con los estilos parentales: convivencia, número de hermanos, edad y género (Furman \& Lanthier, 2002).

Tanto los adolescentes españoles (ES) como los portugueses (PT) participantes en el estudio conviven, en su mayoría, con ambos progenitores, si bien el porcentaje es mayor en los españoles (Ambos padres PT $=79 \%$, ES $=87.9$ $\%, p \leq 0.05$ ); (Padre PT $=1.8 \%$, ES $=2.5 \%$, $p>0.05$ ); (Madre PT $=14.4 \%, \mathrm{ES}=8.1 \%, p$ $\leq 0.05) ;($ Abuelos PT $=1.2 \%, \mathrm{ES}=1.3 \%, p$ $>0.05) ;($ Otros PT $=3.6 \%, \mathrm{ES}=0.2 \%, p \leq$ 0.05), $\left(\chi^{2}(4)=29.86, p<0.001\right)$.

En relación con el tamaño de la familia (número de hermanos por familia), las medias son muy similares: $\mathrm{M}_{\mathrm{PT}}=2.21$ (DT 0.89), $\mathrm{M}_{\mathrm{ES}}$ $=2.27(\mathrm{DT} 1.03), t=1.007, p=0.314$, aunque, entre los adolescentes portugueses existe un mayor porcentaje de hijos/as únicos/as y menor porcentaje con un solo hermano/a (Hno), donde no hay ninguna otra diferencia en función de la nacionalidad y el número de hermanos (Hijo único: $\mathrm{PT}=18.9 \%, \mathrm{ES}=11.1 \%, p \leq 0.05) ;(1$ Hno: $\mathrm{PT}=56 \%, \mathrm{ES}=62.9 \%, p \leq 0.05) ;(2$ Hnos: PT $=17 \%, \mathrm{ES}=18.5 \%, p>0.05) ;(\geq 3$ Hnos PT $=8.1 \%, \mathrm{ES}=7.5 \%, p>0.05),\left(\chi^{2}(1)\right.$ $=14.53, p=0.002)$. Los adolescentes españoles y portugueses fueron comparables en cuanto a su edad: $t(1257)=1.007 p=0.071, r=0.03$, y a la distribución por género: $\chi^{2}(1)=7.95, p=0.337$.

\section{Afecto y comunicación frente a crítica rechazo}

En relación con la percepción que los adolescentes portugueses y españoles tienen sobre el afecto, el interés y la comunicación que manifiestan sus padres, la Manova muestra que los adolescentes españoles logran puntuaciones significativamente mayores que los portugueses en el factor Afecto-comunicación padre, $F(1$, $1250)=9, p=0.003, \eta=0.01$, e inferiores en el factor Crítica rechazo padre, $F(1,1250)=11.6$, $p=0.001, \eta=0.013$, y Crítica rechazo madre, $F(1,1250)=7.49, p=0.006, \eta=0.01$.

En cuanto a las diferencias por género, se encontraron diferencias significativas a favor de los varones en el factor Afecto-comunicación padre, $F(1,1248)=7.26, p=0.007, \eta=$ 0.008 , y Crítica rechazo madre, $F(1,1247)=$ $6.28, p=0.022, \eta=0.006$, si bien la diferencia en el factor Afecto-comunicación padresolo fue hallada entre los adolescentes portugueses, por lo que se encontró una interacción significativa entre los factores Nacionalidad/género en el factor Afecto-comunicación padre, $F(1,1248)=$ 7.04, $p=0.007, \eta=0.007$ (Tabla 1$)$

\section{Tabla 1}

Estadísticos descriptivos de los factores de la escala de afecto (EA-H)

\begin{tabular}{cccccccc}
\hline \multirow{2}{*}{ Scale EA-H } & \multirow{2}{*}{ Nacionalidad } & \multicolumn{2}{c}{ Chicas } & \multicolumn{2}{c}{ Chicos } & \multicolumn{2}{c}{ Total } \\
\cline { 3 - 8 } Afecto comunicación & & $M$ & $D T$ & $M$ & $D T$ & $M$ & $D T$ \\
\hline padre & Española & 37.03 & 9.31 & 37.77 & 9.41 & 37.44 & 9.17 \\
& Portuguesa & 34 & 11.05 & 37.2 & 9.97 & 35.51 & 10.66 \\
& Total & 35.44 & 10.03 & 37.55 & 9.53 & 36.49 & 10.37 \\
\hline \multirow{4}{*}{ Crítica rechazo padre } & Española & 16.94 & 6.79 & 18.75 & 7.67 & 17.9 & 7.22 \\
& Portuguesa & 19.56 & 7.97 & 19.52 & 7.72 & 19.52 & 7.8 \\
& Total & 18.54 & 7.64 & 19.13 & 7.69 & 18.84 & 7.67 \\
\hline \multirow{2}{*}{ Afecto comunicación } & Española & 41.42 & 7.59 & 40.44 & 7.91 & 40.86 & 7.78 \\
madre & Portuguesa & 40.29 & 9.13 & 39.91 & 9.51 & 40.11 & 9.3 \\
& Total & 40.73 & 8.57 & 40.17 & 8.74 & 40.45 & 8.66 \\
\hline \multirow{3}{*}{ Crítica rechazo madre } & Española & 16.95 & 6.39 & 18.42 & 6.61 & 17.79 & 6.55 \\
& Portuguesa & 18.85 & 7.38 & 19.61 & 7.81 & 19.2 & 7.58 \\
& Total & 17.97 & 6.92 & 18.93 & 7.12 & 18.57 & 7.17 \\
\hline & & & & & & & \\
\hline
\end{tabular}

Además, se llevó a cabo una comparación de medias para muestras relacionadas para la variables afecto/comunicación y crítica/rechazo, donde se encontró que, tanto los adolescentes españoles como portugueses, perciben un mayor afecto y mejor comunicación por parte de sus madres que de sus padres, pues no se hallaron diferencias en relación con la actitud crítica y de rechazo de sus padres (Tabla 2 ). 
Tabla 2

Comparación de medias para muestras relacionadas en las variables afecto/comunicación y crítical rechazo

\begin{tabular}{|c|c|c|c|c|c|c|c|}
\hline Nacionalidad & Escala EA-H & & $N$ & $D E$ & $t$ & $p$ & $r$ \\
\hline \multirow{2}{*}{ Portuguesa } & F1 EA afecto/comunicación & $\begin{array}{l}\text { Padre } \\
\text { Madre }\end{array}$ & $\begin{array}{l}35.23 \\
39.98\end{array}$ & $\begin{array}{l}9.44 \\
9.29\end{array}$ & -11.791 & 0.00 & 0.43 \\
\hline & F2 EA crítica/rechazo & $\begin{array}{l}\text { Padre } \\
\text { Madre }\end{array}$ & $\begin{array}{l}19.59 \\
19.28\end{array}$ & $\begin{array}{l}7.82 \\
7.54\end{array}$ & 1.229 & 0.22 & 0.05 \\
\hline \multirow{2}{*}{ Española } & F1 EA afecto/comunicación & $\begin{array}{l}\text { Padre } \\
\text { Madre }\end{array}$ & $\begin{array}{l}37.27 \\
40.89 \\
\end{array}$ & $\begin{array}{l}9.44 \\
9.29\end{array}$ & -9.547 & 0.00 & 0.4 \\
\hline & F2 EA crítica/rechazo & $\begin{array}{l}\text { Padre } \\
\text { Madre }\end{array}$ & $\begin{array}{l}17.89 \\
17.71\end{array}$ & $\begin{array}{l}7.28 \\
6.51\end{array}$ & 0.622 & 0.534 & 40.03 \\
\hline
\end{tabular}

\section{Prácticas parentales (cumplimiento de las normas)}

Con relación a la percepción que los adolescentes portugueses y españoles tienen sobre la forma de establecer y exigir el cumplimiento de las normas de sus padres (Inductiva, indulgente o rígida), la Manova muestra que los adolescentes portugueses logran puntuaciones (Tabla 3) significativamente mayores que los españoles en el factor Forma Rígida Padre, $F(1,1248)=7.09$, $p=0.008, \eta=0.009 ;$ Forma Rígida Madre, $F(1$, 798) $=55.49, p=0.019, \eta=0.007$; Forma Indulgente, $F(1,1247)=53.57, p<0.001, \eta=$ 0.063 , y Forma Indulgente Madre, $F(1,1249)=$ $63.24, p<0.001, \eta=0.073$. Las comparaciones múltiples muestran diferencias significativas en relación con el género en los factores Forma Indulgente Padre, $F(1,1250)=9.64, p=0.002$, $\eta=0.012$, y Forma Indulgente Madre, $F(1$, 1250) $=4.02, p=0.045, \eta=0.005$, en el sentido en que los varones perciben el estilo educativo de sus padres como más indulgente que las mujeres, aunque, estas diferencias solo son evidentes entre adolescentes portugueses. Además, en la interacción nacionalidad/género, solo se encontraron diferencias significativas en el factor Forma Indulgente Padre, $F(1,1245)=$ $8.08, p=0.005, \eta=0.009$.
Tabla 3

Estadísticos descriptivos de los factores de escala normas y exigencias (ENE-H)

\begin{tabular}{cccccccc}
\hline \multirow{2}{*}{ Escala ENE } & \multirow{2}{*}{ Nacionalidad d } & \multicolumn{2}{c}{ Chicas } & \multicolumn{2}{c}{ Chicos } & \multicolumn{2}{c}{ Total } \\
\cline { 3 - 8 } Forma inductiva & & $M$ & $D E$ & $M$ & $D E$ & $M$ & $D E$ \\
Padre & Española & 37.03 & 9.19 & 37.77 & 9.73 & 37.44 & 9.49 \\
& Portuguesa & 36.06 & 10.27 & 37.45 & 12.56 & 36.71 & 11.41 \\
& Total & 36.45 & 9.85 & 37.61 & 11.26 & 37.04 & 10.6 \\
\hline \multirow{2}{*}{ Forma rígida } & Española & 28.45 & 7.8 & 28.33 & 8.15 & 28.45 & 7.8 \\
Padre & Portuguesa & 29.82 & 8.05 & 29.47 & 9.19 & 30 & 8.4 \\
& Total & 29.14 & 7.81 & 28.91 & 8.71 & 29.03 & 8.27 \\
\hline \multirow{2}{*}{$\begin{array}{c}\text { Forma indulgente } \\
\text { Padre }\end{array}$} & Española & 17.82 & 5.3 & 18.05 & 6.09 & 17.7 & 5.66 \\
& Portuguesa & 19.58 & 5.28 & 21.94 & 6.98 & 20.7 & 6.25 \\
\multirow{2}{*}{ Forma inductiva } & Total & 18.71 & 5.35 & 19.83 & 6.8 & 19.52 & 6.29 \\
Madre & Española & 38.87 & 8.05 & 38.51 & 8.44 & 38.67 & 8.26 \\
& Portuguesa & 38.65 & 8.43 & 37.4 & 9.23 & 38.07 & 8.83 \\
& Total & 38.74 & 8.27 & 37.94 & 8.86 & 38.34 & 8.58 \\
\hline \multirow{2}{*}{ Forma rígida } & Española & 29.35 & 7.14 & 29.43 & 8.25 & 29.38 & 7.74 \\
Madre & Portuguesa & 31.25 & 7.99 & 30.28 & 8.96 & 30.77 & 8.36 \\
& Total & 30.49 & 7.71 & 29.86 & 8.62 & 30.17 & 8.19 \\
\hline \multirow{2}{*}{ Forma indulgente } & Española & 18 & 4.95 & 18.24 & 6.03 & 18.05 & 5.49 \\
Madre & Portuguesa & 20.73 & 6.05 & 22.18 & 6.94 & 21.42 & 6.52 \\
& Total & 19.53 & 5.78 & 20.12 & 6.71 & 19.99 & 6.27 \\
\hline
\end{tabular}

Asimismo, se realizó una comparación de medias para muestras relacionadas para las variables Forma Inductiva-RígidaIndulgentepadre y madre, donde se encontró que los adolescentes portugueses y españoles perciben las prácticas parentales de sus madres como más inductivas y rígidas que las de sus padres (Tabla 4).

\section{Tabla 4}

Comparación de medias para muestras relacionadas en las variables estilo inductivo-rígido-indulgente padre y madre

\begin{tabular}{cccccccc}
\hline \multirow{2}{*}{ Escala ENE } & \multirow{2}{*}{ Nacionalidad } & \multicolumn{2}{c}{ Chicas } & \multicolumn{2}{c}{ Chicos } & \multicolumn{2}{c}{ Total } \\
\cline { 3 - 8 } & & $M$ & $D E$ & $M$ & $D E$ & $M$ & $D E$ \\
\hline \multirow{2}{*}{$\begin{array}{c}\text { Forma inductiva } \\
\text { Padre }\end{array}$} & Española & 37.03 & 9.19 & 37.77 & 9.73 & 37.44 & 9.49 \\
& Portuguesa & 36.06 & 10.27 & 37.45 & 12.56 & 36.71 & 11.41 \\
& Total & 36.45 & 9.85 & 37.61 & 11.26 & 37.04 & 10.6 \\
\hline \multirow{2}{*}{ Forma rígida } & Española & 28.45 & 7.8 & 28.33 & 8.15 & 28.45 & 7.8 \\
Padre & Portuguesa & 29.82 & 8.05 & 29.47 & 9.19 & 30 & 8.4 \\
& Total & 29.14 & 7.81 & 28.91 & 8.71 & 29.03 & 8.27 \\
\hline \multirow{2}{*}{ Forma indulgente } & Española & 17.82 & 5.3 & 18.05 & 6.09 & 17.7 & 5.66 \\
Padre & Portuguesa & 19.58 & 5.28 & 21.94 & 6.98 & 20.7 & 6.25 \\
& Total & 18.71 & 5.35 & 19.83 & 6.8 & 19.52 & 6.29 \\
\hline \multirow{2}{*}{ Forma inductiva } & Española & 38.87 & 8.05 & 38.51 & 8.44 & 38.67 & 8.26 \\
Madre & Portuguesa & 38.65 & 8.43 & 37.4 & 9.23 & 38.07 & 8.83 \\
& Total & 38.74 & 8.27 & 37.94 & 8.86 & 38.34 & 8.58 \\
\hline \multirow{2}{*}{ Forma rígida } & Española & 29.35 & 7.14 & 29.43 & 8.25 & 29.38 & 7.74 \\
Madre & Portuguesa & 31.25 & 7.99 & 30.28 & 8.96 & 30.77 & 8.36 \\
& Total & 30.49 & 7.71 & 29.86 & 8.62 & 30.17 & 8.19 \\
\hline \multirow{2}{*}{$\begin{array}{c}\text { Forma indulgente } \\
\text { Madre }\end{array}$} & Española & 18 & 4.95 & 18.24 & 6.03 & 18.05 & 5.49 \\
& Portuguesa & 20.73 & 6.05 & 22.18 & 6.94 & 21.42 & 6.52 \\
\hline & Total & 19.53 & 5.78 & 20.12 & 6.71 & 19.99 & 6.27 \\
\hline
\end{tabular}




\section{Prácticas parentales frente a afecto/ comunicación}

Para determinar en qué medida la práctica parental se relaciona con la percepción que los adolescentes tienen sobre el afecto y la comunicación que manifiestan sus padres, se llevaron a cabo varios análisis de regresión lineal múltiple de un solo modelo, en los que las variables dependientes fueron afecto comunicación madre o afecto comunicación padre,donde se incluyeron en todos los modelos como variables independientes los factores de la escala ENE-h, padre Forma Inductiva-RígidaIndulgente y madre Forma Inductiva-RígidaIndulgente. Los análisis de regresión se realizaron por separado, uno para la muestra portuguesa y otro para la muestra española.

Con el fin de evitar posibles sesgos derivados de las diferencias encontradas en la variable convivencia entre adolescentes portugueses y españoles, se decidió realizar los modelos de regresión solo con el grupo de participantes que convive con ambos progenitores.

\section{Modelo de Regresión Lineal EA Afecto- comunicación padre}

Este análisis reveló que el modelo predictivo del afecto y la comunicación padre en la muestra española explica un $42.4 \%$ de la varianza, donde es significativo, $F(6,461)=$ $50.578, p<0.001$, y en la muestra portuguesa explica un $43.2 \%$ de la varianza,donde resulta significativo, $F(6,521)=64.563, p<0.001$. En la Tabla 5 se puede observar que las variables con poder predictivo, tanto en la muestra española como en la portuguesa, son Forma Inductiva e Indulgente padre y Forma Indulgente madre, y solo en la muestra española Forma Rígida madre, aunque la variable con un mayor poder predictivo en ambas muestras es Forma Inductiva padre. La principal diferencia entre adolescentes españoles y portugueses en la capacidad predictiva que los estilos parentales tienen del afecto y comunicación con su padre se aprecia en relación con la variable Forma Rígida madre,yaque esta solo tiene poder predictivo, lineal inverso, entre los adolescentes españoles.

\section{Tabla 5}

Coeficientes de regresión parcial. VD: F1 EA padre Afecto-comunicación

\begin{tabular}{|c|c|c|c|c|c|c|c|c|c|}
\hline \multirow{2}{*}{ Nacionalidad } & \multirow{2}{*}{\multicolumn{2}{|c|}{ Variables predictora }} & \multicolumn{2}{|c|}{$\begin{array}{l}\text { Coeficientes no } \\
\text { estandarizados }\end{array}$} & \multirow{2}{*}{$\begin{array}{c}\begin{array}{c}\text { Coeficientes } \\
\text { tipificados }\end{array} \\
\text { Beta }\end{array}$} & \multirow[t]{2}{*}{$t$} & \multirow[t]{2}{*}{$p$} & \multicolumn{2}{|c|}{ Colinealidad } \\
\hline & & & & & & & & Tolerancia & VIF \\
\hline \multirow{7}{*}{$\begin{array}{l}\text { Española } \\
\text { (ES) }\end{array}$} & \multicolumn{2}{|c|}{ Constante } & 17.377 & 2.325 & & 7.473 & 0.00 & & \\
\hline & & Forma inductiva & 0.626 & 0.053 & 0.631 & 11.758 & 0.00 & 0.495 & 2.022 \\
\hline & \multirow{2}{*}{ Padre } & Forma rigida & 0.038 & 0.084 & 0.031 & 0.451 & 0.652 & 0.393 & 2.414 \\
\hline & & Forma indulgente & 0.449 & 0.12 & 0.275 & 3.739 & 0.00 & 0.364 & 2.784 \\
\hline & \multirow{3}{*}{ Madre } & Forma inductiva & -0.026 & 0.06 & -0.023 & -.437 & 0.663 & 0.506 & 1.975 \\
\hline & & Forma rígida & -0.196 & 0.083 & -0.162 & -2.354 & 0.019 & 0.402 & 2.307 \\
\hline & & Forma indulgente & -0.332 & 0.125 & -0.196 & -2.654 & 0.008 & 0.361 & 2.834 \\
\hline \multirow{7}{*}{$\begin{array}{l}\text { Portuguesa } \\
\text { (PT) }\end{array}$} & \multicolumn{2}{|c|}{ Constante } & 12.622 & 1.968 & & 6.412 & 0.00 & & \\
\hline & \multirow{3}{*}{ Padre } & Forma inductiva & 0.551 & 0.048 & 0.583 & 11.454 & 0.00 & 0.438 & 2.283 \\
\hline & & Forma rígida & 0.026 & 0.066 & 0.021 & 0.394 & 0.694 & 0.409 & 2.442 \\
\hline & & Forma indulgente & 0.22 & 0.088 & 0.13 & 2.497 & 0.013 & 0.416 & 2.403 \\
\hline & \multirow{3}{*}{ Madre } & Forma inductiva & 0.069 & 0.058 & 0.056 & 1.184 & 0.237 & 0.505 & 1.981 \\
\hline & & Forma rígida & -0.011 & 0.069 & -0.009 & -0.162 & 0.871 & 0.393 & 2.541 \\
\hline & & Forma indulgente & -0.233 & 0.089 & -0.138 & -2.614 & 0.009 & 0.408 & 2.453 \\
\hline & \multicolumn{9}{|c|}{$\begin{array}{l}\text { Nota. ES }\left(R^{2} \text { corregida }=0.424 ; \text { Durbin }\right. \\
\text { Watson }=2.019) ; \text { PT }\left(R^{2} \text { corregida }\right. \\
=0.432 ; \text { Durbin-Watson }=1.838)\end{array}$} \\
\hline
\end{tabular}

Modelo de Regresión Lineal EA Afectocomunicación madre

El análisis reveló que el modelo predictivo del afecto y la comunicación madre en la muestra española explica un $32.4 \%$ de la varianza,y es significativo, $F(6,461)=33.339, p<0.001$, y en la muestra portuguesa explica un $33.8 \%$ de la varianza, e igualmente es significativo, $F(6$, $521)=43.473, p<0.001$. La Tabla 6 muestra que el estilo parental con mayor poder predictivo sobre el afecto y la comunicación de la madre es Forma Inductiva madre, tanto en españoles como en portugueses. Además, el análisis de regresión muestra la existencia de diferencias en función de la nacionalidad en el efecto del estilo parental paterno en el afecto y comunicación de la madre. Si bien, entre adolescentes españoles, la Forma Inductiva padre predice el afecto y comunicación madre, entre los adolescentes portugueses son Forma Rígida e Indulgente padre las que tienen poder predictivo. 
Tabla 6

Coeficientes de regresión parcial. VD: F1 EA madre Afecto-comunicación

\begin{tabular}{|c|c|c|c|c|c|c|c|c|c|}
\hline \multirow{2}{*}{ Nacionalidad } & \multirow{2}{*}{\multicolumn{2}{|c|}{ Variables predictora }} & \multicolumn{2}{|c|}{$\begin{array}{l}\text { Coeficientes no } \\
\text { estandarizados }\end{array}$} & \multirow{2}{*}{$\begin{array}{c}\begin{array}{c}\text { Coeficientes } \\
\text { tipificados }\end{array} \\
\text { B }\end{array}$} & \multirow[t]{2}{*}{$t$} & \multirow{2}{*}{$p$} & \multicolumn{2}{|c|}{ Colinealidad } \\
\hline & & & B & ET & & & & Tolerancia & VIF \\
\hline \multirow{7}{*}{$\begin{array}{l}\text { Española } \\
\text { (ES) }\end{array}$} & \multicolumn{2}{|c|}{ Constante } & 20.558 & 2.136 & & 9.623 & 0.00 & & \\
\hline & \multirow{4}{*}{ Padre } & Forma inductiva & 0.101 & 0.049 & 0.12 & 2.042 & 0.042 & 0.482 & 2.073 \\
\hline & & Forma rigida & 0.092 & 0.077 & 0.09 & 1.196 & 0.232 & 0.394 & 2.405 \\
\hline & & Forma indulgente & 0.035 & 0.11 & 0.025 & 0.318 & 0.751 & 0.362 & 2.817 \\
\hline & & Forma inductiva & 0.448 & 0.057 & 0.456 & 7.83 & 0.00 & 0.493 & 2.028 \\
\hline & \multirow[t]{2}{*}{ Madre } & Forma rigida & -0.228 & 0.077 & -0.221 & -2.974 & 0.003 & 0.402 & 2.306 \\
\hline & & Forma indulgente & 0.141 & 0.115 & 0.099 & 1.23 & 0.22 & 0.359 & 2.859 \\
\hline \multirow{7}{*}{$\begin{array}{l}\text { Portuguesa } \\
\text { (PT) }\end{array}$} & \multicolumn{2}{|c|}{ Constante } & 17.72 & 1.84 & & 9.633 & 0.00 & & \\
\hline & \multirow{3}{*}{ Padre } & Forma inductiva & 0.032 & 0.045 & 0.039 & 0.708 & 0.479 & 0.442 & 2.265 \\
\hline & & Forma rigida & -0.162 & 0.062 & -0.148 & -2.611 & 0.009 & 0.411 & 2.431 \\
\hline & & Forma indulgente & 0.174 & 0.082 & 0.120 & 2.118 & 0.035 & 0.416 & 2.402 \\
\hline & \multirow{3}{*}{ Madre } & Forma inductiva & 0.608 & 0.054 & 0.574 & 11.276 & 0.00 & 0.512 & 1.954 \\
\hline & & Forma rigida & 0.08 & 0.064 & 0.072 & 1.25 & 0.212 & 0.399 & 2.509 \\
\hline & & Forma indulgente & -0.154 & 0.083 & -0.106 & -1.865 & 0.063 & 0.41 & 2.44 \\
\hline & \multicolumn{9}{|c|}{$\begin{array}{l}\text { Nota.ES }\left(R^{2} \text { corrected }=0.324 ; \text { Durbin- }\right. \\
\text { Watson }=1.875) ; \text { PT }\left(R^{2} \text { corrected }\right. \\
\quad=0.338 ; \text { Durbin-Watson }=1.704)\end{array}$} \\
\hline
\end{tabular}

\section{Discusión}

En esta investigación se presenta una comparación entre dos poblaciones limítrofes, Extremadura en España y Alentejo en Portugal, sobre las pautas o estilos de crianza, medidas a través de la percepción de los adolescentes sobre el afecto, comunicación y las actitudes críticas y de rechazo de sus progenitores.

En relación con el primer objetivo, en las comparaciones de la percepción de los adolescentes en función de la nacionalidad, los padres españoles obtienen mayores puntuaciones en Afecto-comunicación, y menores en crítica, rechazo y Forma Rígida e Indulgente que los portugueses, lo que indicaría que, si bien el estilo de crianza de los hijos está determinado por múltiples factores (Belsky \& Jaffee, 2006; Ramírez, 2005), es un fenómeno sujeto especialmente a las particularidades de las pautas culturales en las que se vive (Darling \& Steinberg, 1993).

En cuanto a las diferencias en función del género de los hijos, si bien, entre los adolescentes españoles no se han hallado diferencias entre varones y mujeres con relación al Afectocomunicación percibido de sus padres, los chicos españoles perciben a sus padres como más críticos, y se sienten más rechazados por ellos que las chicas españolas. Esto indicaría que los chicos españoles son más sensibles que las chicas al control, la supervisión, la autoridad, la hostilidad y la permisividad, tanto de las madres como de los padres (Rodríguez, del Barrio, \& Carrasco, 2009). Además, los chicos perciben unas pautas educativas más negativas y centradas en la irritabilidad, rechazo, castigos, riñas y negligencia (León et al., 2015; Mestre, Samper, Nácher, Tur, $\&$ Cortés, 2007), y en la etapa de la adolescencia suelen tener más conflictos con sus padres que las chicas (Motrico et al., 2001).

Respecto a los adolescentes portugueses, los varones perciben un mayor afecto $y$ comunicación de su padre, y mayor críticarechazo de su madre, que las chicas. Según Motrico et al. (2001), los adolescentes discuten más con sus madres que con sus padres. Además, las madres suelen emplear un mayor número de comportamientos de control, exigencias y estrategias de disciplina (Mendo et al., 2019; Rodríguez et al., 2009). Esto coincide con otros estudios, como los de Laible y Carlo (2004), que encuentran que los adolescentes consideran a sus madres como más implicadas y controladoras que a sus padres.

Además, los resultados de las comparaciones de medias para muestras relacionadas entre padres y madres, tanto en adolescentes españoles como en los portugueses, constatan que la educación paterna y materna es percibida de forma diferente por los hijos (Molina, Raimundi, $\&$ Bugallo, 2017). Concretamente, por un lado, las prácticas educativas de las madres son percibidas como más positivas que las de los padres, ya que éstas logran mayores puntuaciones en afecto y menores en rechazo (Bersabé et al., 2001; García, Cerezo, De la Torre, Villa, \& Casanova, 2011). Por otro lado, las madres son percibidas como más inductivas y rígidas que los padres, donde se hace patente que la comunicación y el afecto no solo no están reñidos con la exigencia y el control (Torío, Peña, \& Rodríguez, 2008), sino que —al menos en cuanto el estilo parental materno- parecen estar claramente relacionados.

En este sentido, muchas investigaciones ponen de manifiesto la mayor implicación de las madres frente a los padres en las prácticas de crianza. Laible y Carlo (2004) comprueban que las 
madres son más afectuosas y favorecen más la autonomía de los hijos. Tur-Porcar et al. (2012), con una muestra de 2788 alumnos de 10-15 años, confirman diferencias significativas entre la crianza ejercida por las madres y la ejercida por los padres, donde encuentran que las madres mantienen una mayor influencia sobre los hijos, independientemente del sexo de los mismos.

No obstante, diferentes estudios informan de una atenuación de las diferencias en los cuidados parentales según el género de los progenitores (Lanz, Scabini, Vermulst, \& Gerris, 2001; Mendo et al., 2019; Pereira, Cannavarro, Cardoso, \& Mendoça, 2009). Esta tendencia, aunque en Portugal ha comenzado antes, parece ser progresiva en ambos países (Alcañiz \& Guerreiro, 2009).

Estas diferencias entre padre y madre se relacionan con un mayor tiempo de interacción con los hijos/as por parte de las madres que de los padres (García et al., 2011), lo que explicaría el no haber hallado diferencias en el estilo indulgente entre progenitores, caracterizado por la ausencia de normas y límites, lo que reduce la necesidad de interacciones entre los padres/ madres y sus hijos/as.

En relación con nuestro segundo objetivo, los resultados de los análisis de regresión manifiestan que la Forma Inductiva que tienen los padres de establecer y exigir el cumplimiento de las normas, caracterizada por el uso de la razón y explicaciones a los hijos de las consecuencias del incumplimiento de las normas y de las acciones prohibidas, es un fuerte predictor del afecto y cariño de los progenitores españoles y portugueses. También, en ambas muestras los datos revelan que la Forma Indulgente, caracterizada por la ausencia de normas y límites a la conducta de los hijos, es un factor relevante cuando se trata de predecir el afecto y comunicación de los padres, pero no el de las madres (Bersabé et al., 2001).

Además, los análisis revelan que la forma en que uno de los progenitores establece y exige el cumplimiento de las normas predice el cariño que los hijos perciben del otro progenitor, especialmente una forma que evite la imposición de normas y un nivel alto de exigencias (la
Forma Rígida madre y padre tiene un poder predictivo, lineal inverso, sobre la variable Afecto-comunicación).

En definitiva, se percibe afecto y comunicación cuando existe coincidencia y consistencia en los comportamientos de ambos progenitores, que cuando no se presenta, provoca rechazo y problemas emocionales y conductuales en los hijos (Rodríguez et al., 2009).

Por último, respecto a la coincidencia $-\mathrm{O}$ no - de los diferentes factores como predictores en ambas muestras de adolescentes españoles y portugueses, en relación con el modelo predictivo de las variables afecto y comunicación madre, encontramos que para los adolescentes españoles, la Forma Inductiva de la madre y del padre predice el afecto y la comunicación, mientras que, entre los adolescentes portugueses es la Forma Inductiva de la madre y la Forma Indulgente del padre las que tienen poder predictivo, lo que corrobora nuevamente que los estilos de crianza pueden tener efectos distintos al relacionarlos con la cultura (Darling \& Steinberg, 1993).

La principal limitación del estudio —además del uso exclusivo de autoinformes para la recogida de información, o el diseño transversal del estudio que dificulta el establecimiento de relaciones de causalidad entre variableses que dentro de un modelo bidireccional de las relaciones padres-hijos, en el que las características de los hijos y de los padres son clave para la comprensión de los factores que determinan los estilos de crianza, se ha recogido solo la percepción de los hijos como una única fuente de información. Además, los resultados deben ser analizados con cautela a la hora de ser extrapolarlos a otros contextos.

No obstante, y a pesar de algunas limitaciones, se confirma la existencia de diferencias intraculturales e interculturales en la percepción del afecto, el interés y comunicación, y en la forma de establecer y exigir el cumplimiento de las normas que manifiestan los padres españoles y portugueses. Además, el estudio permite ampliar el conocimiento sobre la asociación de los estilos de parentales con el afecto y la comunicación percibido por los hijos 
Finalmente, y teniendo en cuenta que actualmente es muy común que ambos padres trabajen, lo que, en general, conlleva una reducción del tiempo de convivencia entre los miembros de las familias y, en particular, un tiempo de dedicación a los hijos propio de determinados patrones de crianza, se considera importante investigar las relaciones entre los estilos parentales y las diferentes políticas de conciliación familiar que pudieran contribuir a un contacto más adecuado y de calidad entre padres e hijos, que afecte los vínculos y la estabilidad familiar, y por ende el estilo parental.

\section{Referencias}

Alcañiz, M., \& Guerreiro, M. D. (2009). Tiempos, trabajos e identidades. Análisis comparativo entre mujeres españolas y portuguesas. Aparkia, 20, 123-145.

Alegre, A. (2011). Parenting Styles and Children 's emotional intelligence: What do we know? The family Journal: Counseling and therapy for couples and families, 19(1), 56-62.

Aroca, C., \& Cánovas, L. P. (2012). Los estilos educativos parentales desde los modelos interactivos y de construcción conjunta: Revisión de las investigaciones. Teoría De La Educación, 24(2), 149-176.

Baumrind, D. (1971). Current patterns of parental authority. Developmental Psychology Monograph, 4, 1-103.

Baumrind, D. (1972). An exploratory study of socialization effects on black children: Some black-white comparisons. Child Development, 43(1), 261-267.

Baumrind, D. (1996). The Discipline Controversy Revisted. Family Relations, 4(4), 405-414.

Belsky, J., \& Jaffee, S. R. (2006). The multiple determinants of parenting. En D. Cicchetti \& C. J. Donald (Eds.), Developmental psychopathology: Risk, disorder, and adaptation (Vol. 3,pp. 38-85). Hoboken, NJ: Wiley.

Bersabé, R., Fuentes, M. J., \& Motrico, E. (2001). Análisis Psicométrico de dos escalas para evaluar estilos educativos parentales. Psicothema, 13(4), 678-684.

Darling, N., \& Steinberg, L. (1993). Parenting style as context: An integrative model. Psychological Bulletin, 113(3), 487-496.

Domenech, M. M., Donovick, M. R., \& Crowley, S. L. (2009). Parenting styles in a cultural context: observations of "protective parenting" in first-generation Latinos. Family Process, 48(2), 195-210.

Dwairy, M. A. (2008). Parental inconsistency versus parental authoritarianism: Associations with symptoms of psychological disorders. Journal of Youth and Adolescence, 37(5), 616-626. https://doi.org /10.1007/s10964-007-9169-3

Fuentes, M. C., García, F., Gracia, E., \& Alarcón, A. (2015). Los estilos parentales de socialización y el ajuste psicológico. Un estudio con adolescentes españoles. Revista de Psicodidáctica, 20(1), 117-138.

Furman, W., \& Lanthier, R. (2002). Parenting siblings. En M. H. Bornstein (Ed.), Handbook of Parenting: Children and Parenting (Vol. 1, pp. 165-188). Mahwah, NJ: Lawrence Erlbaum Associates.

García, F., \& Gracia, E. (2010). ¿Qué estilo de socialización parental es el idóneo en España? Un estudio con niños y adolescentes de 10 a 14 años. Infancia y Aprendizaje, 33(3),365-384.

García, M., Cerezo, M., De la Torre, M., Villa, M., $\&$ Casanova, P. (2011). Prácticas educativas paternas y problemas internalizantes y externalizantes en adolescentes españoles. Psicothema, 23(4), 654-659.

García, O. F., Serra, E., Zacarés, J. J., \& García, F. (2018). Parenting styles and shortand long-term socialization outcomes: A study among Spanish adolescents and older adults. Psychosocial Intervention, 27(3), 153-161. https://doi.org/10.5093/pi2018a2 1

González, M., \& Landero, R. (2012). Diferencias en la percepción de estilos parentales entre jóvenes y adultos de la misma familia. Summa Psicológica UST, 9(1), 53-64. 
Gracia, E., Lila, M., \& García., F (2008). Estilos educativos parentales y ajuste psicológico de los hijos: Cuestionando la preeminencia del estilo autoritativo. Ponencia presentada en el $V$ Congreso Internacional de Psicología y Educación. Oviedo, España.

Grolnick, W., \& Pomerantz, E. (2009). Issues and challenges in studying parental control: Toward a new conceptualization. Child Development Perspectives, 3(3), 165-170.

Hadzic, R., Magee, C. A., \& Robinson, L. (2013). Parental employment and child behaviors: Do parenting practices underlie these relationships? International Journal of Behavioral Development, 37(4), 332-339.

Huertas, I. P. M., \& Rego, C. (2005). La importancia del capital humano en Alentejo y Extremadura. Papeles de Economía Española, 21, 101-111.

Instituto Nacional de Estadística. (2014). La Península Ibérica en cifras 2014. Madrid: Autor.

Jiménez, M. Á., \& Fernández, J. I. (2009). El papel del catastro en el proyecto Otalex: una experiencia "Inspiradora". CT: Catastro, 66, 47-63

Laible, D. J., \& Carlo, G. (2004). The differential relations of maternal and paternal support and control to adolescent social competent, self-worth, and sympathy. Journal of Adolescent Research, 19, 759-782. https://do i.org/10.1177/0743558403260094

Lanz, M., Scabini, E., Vermulst, A. A., \& Gerris, J. R. (2001). Congruence on child rearing in families with early adolescent and middle adolescent children. International Journal of Behavioral Development, 25(2), 133-139.

León, B., Fajardo, F., Mendo, S., Rasskin, I., \& Iglesias, D. (2018). Impact of the familiar environment in 11-14-year-old minors' mental health. International journal of environmental research and public health, $15(7), 1314$.

León, B., Felipe, E., Polo, M. I., \& Fajardo. F. (2015). Aceptación-rechazo parental y perfiles de victimización y agresión en situaciones de bullying. Anales de Psicología, $31(2), 600-606$.
León, B., Mendo, S., Polo, M. I., \& López, V. M. (2019). Parental Psychological Control and Emotional and Behavioral Disorders among Spanish Adolescents. International journal of environmental research and public health, 16(3), 507.

López-Soler, C., Puerto, J. C., López-Pina, J. A., \& Prieto, M. (2009). Percepción de los estilos educativos parentales e inadaptación en menores pediátricos. Anales de psicología, 25(1), 70-77.

Maccoby, E. E., \& Martin, J. A. (1983). Socialization in the context of the family: Parent-child interaction. En E. M. Hetherington \& P. H. Mussen (Eds.), Handbook of Child Psychology Socialization, Personality, and Social Development (Vol. 4, pp. 1-101).Nueva York, NY: Wiley.

Mansager, E., \& Volk, R. (2004). Parent's prism. Three dimensions of effective parenting. Journal of Individual Psychology, 60(3), 277-293.

Martínez, J. L., Fuertes, A., Ramos, M., \& Hernández, A. (2003). Consumo de drogas en la adolescencia: Importancia del afecto y la supervisión parental. Psicothema, 15(2), 161-166.

Mendo, S., León, B., Polo, M. I., Yuste, R., \& López, V. M. (2019). The Role of Parental Acceptance-Rejection in Emotional Instability During Adolescence. International journal of environmental research and public health, 16(7), 1194.

Mestre, M. V., Tur, A. M., Samper, P., \& Latorre, A. (2010). Inestabilidad emocional y agresividad. Factores predictores. Ansiedad y Estrés, 16(1), 33-45.

Mestre, M. V., Tur, A. M., Samper, P., Nácher, M. J., \& Cortés, M. T. (2007). Estilos de crianza en la adolescencia y su relación con el comportamiento prosocial. Revista latinoamericana de psicología, 39(2), 211-225.

Molina, M. F., Raimundi, M. J., \& Bugallo, L. (2017). La percepción de los estilos de crianza y su relación con las autopercepciones de los niños de Buenos 
Aires: Diferencias en función del género. Universitas Psychologica, 16(1), 1-12.

Moreno, D., Estévez, E., Jiménez, T., \& Murgui, S. (2018). Parenting Style and Reactive and Proactive Adolescent Violence: Evidence from Spain. International Journal of Environmental Research Public Health, 15(12), 2634.

Motrico, E., Fuentes, M. J., \& Bersabé, R. (2001). Discrepancias en la percepción de los conflictos entre padres e hijos/as a lo largo de la adolescencia. Anales de psicología, 17(1), 1-13.

Muris, P., Meesters, C., Morren, M., \& Moorman, L. (2004). Anger and hostility in adolescents: Relationships with self-reported attachment style and perceived parental rearing styles. Journal of Psychosomatic Research, 57(3), 257-264

Nunes, C., Bodden, D., Lemos, I., Lorence, B., \& Jiménez, L. (2014). Prácticas parentales y calidad de vida en adolescentes holandeses y portugueses: un estudio transcultural. Revista de Psicodidáctica, 19(2), 327-346.

Oliva, A., Parra, A., \& Arranz, E. (2008). Estilos relacionales parentales y ajuste adolescente. Infancia y Aprendizaje, 31(1), 93-106.

Oliva, A., Parra, A., Sánchez-Queija, I., \& López, F. (2007). Estilos educativos maternos y paternos: Evaluación y relación con el ajuste del adolescente. Anales de Psicología, 23(3), 49-56.

Pereira, A. I., Cannavarro, C., Cardoso, M. F., \& Mendoça, D (2009). Patterns of Parental Rearing Styles and Child Behavior Problemas among Portugueses SchoolAged Children. Journal of Child and Family Studies, 18(4), 454-464.

Polo, M. I., Fajardo, F., Martín, E., Gómez, T., \& León, B. (2012). Aceptación/rechazo parental en una muestra de adolescentes. Diferencias según sexo y edad. International Journal of Developmental and Educational Psychology, 1(1), 329-336.

Ramírez, M. A. (2005). Padres y desarrollo de los hijos: prácticas de crianza. Estudios Pedagógicos, 31(2), 167-177
Rodrigo, M. J., \& Palacios, J. (1998). Familia y desarrollo humano. Madrid: Alianza Editorial.

Rodrigues, Y., Veiga, F., Fuentes, M. C., \& García, F. (2013). Parenting and adolescents' selfesteem: The Portuguese context. Revista de Psicodidáctica, 18(2), 395-416.

Rodríguez, M. A., del Barrio, V., \& Carrasco, M. A. (2009). Consistencia interparental y su relación con la agresión y la sintomatología depresiva en niños y adolescentes. Revista de Psicopatología y Psicología Clínica, 14(1), 51-60.

Rohner, R. P. (1975). They love me, they love me not: a worldwide study of the effects of parental acceptance-rejection. New Haven, CT: HRAF.

Rohner, R. P., \& Carrasco, M. A. (2014). Teoría de la aceptación-rechazo interpersonal (Ipartheory): bases conceptuales, método y evidencia empírica. Acción Psicológica, 11(2), 9-26.

Rudy, R., \& Grusec, J. E. (2006). Authoritarian parenting in individualist and collectivist groups: Associations with maternal emotion and cognition and children's selfesteem. Journal of Family Psychology, 20(1), 68-78.

Sánchez-Sandoval, Y. (2002). El ajuste de niños y niñas y su vida familiar: Un estudio longitudinal (Tesis Doctoral). Universidad de Sevilla, España.

Serrano, G., El-Astal, S., \& Faro, F. (2004). La adolescencia en España, Palestina y Portugal: análisis comparativo. Psicothema, 16(3), 468-475.

Tabachnick, B., \& Fidell, L. (2007). Using multivariate statistics ( $5^{\mathrm{a}} \mathrm{ed}$.). Boston, MA: Pearson Education.

Torío, S., Peña, J. V., \& Inda, M. (2008). Estilos de educación familiar. Psicothema, 20(1), 62-70.

Torío, S., Peña, J., \& Rodríguez, M. (2008). Estilos educativos parentales: revisión bibliográfica y reformulación teórica. Teoría De La Educación. Revista Interuniversitaria, 20, 151-178. 
Santiago Mendo Lázaro, María-Isabel Polo del Río, Fernando Fajardo Bullón, et al.

Tur-Porcar, A. M., Mestre, M. V., Samper, P., \& Malonda, E. (2012). Crianza y agresividad de los menores: ¿Es diferente la influencia del padre y de la madre? Psicothema, 24(2), 284-298.

\section{Notas}

* Artículo de investigación. 